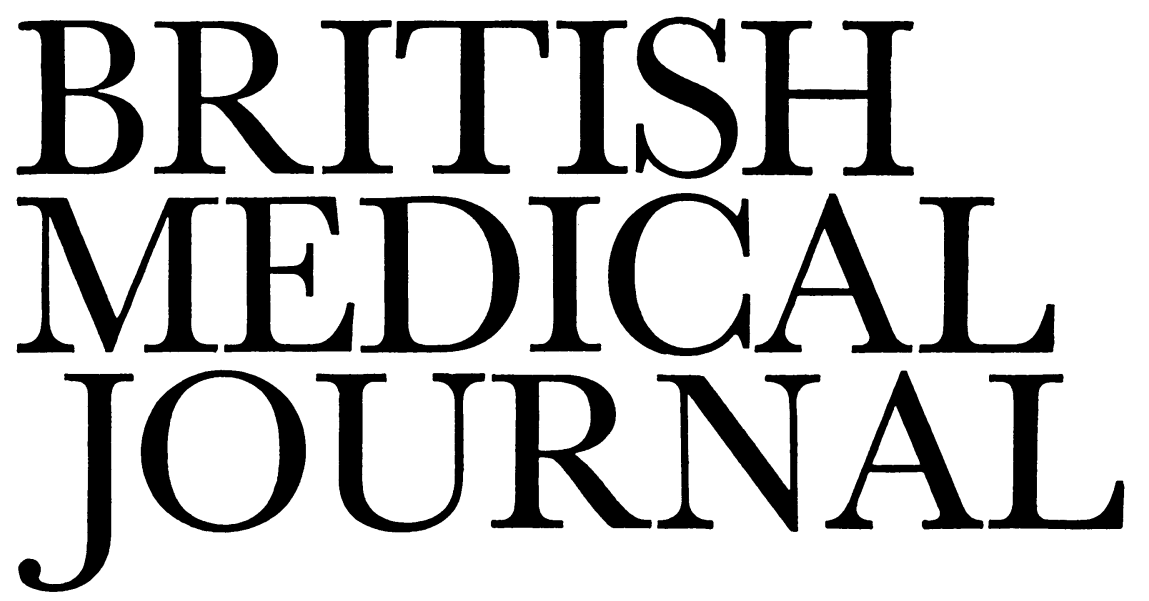

LONDON, SATURDAY 31 JANUARY 1981

\title{
Analgesic nephropathy
}

Is analgesic nephropathy, like smallpox, now only a part of medical history? Did the campaign to remove phenacetincontaining compound analgesic tablets from pharmacists' counters succeed in eliminating this often fatal disorder?

Thirty years ago analgesic nephropathy had not been recognised and most of its victims were diagnosed as having chronic pyelonephritis. After the initial description by Spühler and Zollinger ${ }^{1}$ several larger and carefully analysed series of cases were published, and the clinical and radiological features became defined. ${ }^{2-6}$ Clinicians prepared to take a careful history from the patient and relatives and to examine urine specimens for evidence of analgesic intake made the diagnosis quite frequently.

In Nottingham, a reasonably typical urban community, analgesic nephropathy was responsible for at least $12 \%$ of cases of severe chronic renal failure presenting between 1970 and 1975-around 10 new cases each year from each million population. ${ }^{6}$ In about a third of these patients the diagnosis had been "missed" and came to light only as a result of the epidemiological survey. Statistics from the European Dialysis and Transplant Association ${ }^{7}$ on the causes of chronic renal failure receiving treatment suggest that around $3.1 \%$ are drug-induced, varying from $0.4 \%$ in Spain to $17.5 \%$ in Switzerland, with $1.2 \%$ given as the British figure. These differences have many causes. In addition to misclassification and geographical difference in prevalence some distortion results from the selection of patients for dialysis and transplantation, since those who have misused drugs often have social or psychiatric reasons making them less suitable for treatment. In Scandinavia ${ }^{7}$ and in Scotland ${ }^{8}$ recent data have suggested a drop in the number of cases, and nephrologists in England also have the impression that new patients with analgesic nephropathy are becoming less common in their clinics. These observations may be the result of phenacetin being taken out of most analgesic mixtures after representations by nephrologists during the early 1970s. Concern remains, however, that paracetamol (which was widely substituted for phenacetin) might also be nephrotoxic, especially when in compound analgesic tablets. Indeed, some nephrologists suggest that most common analgesics, even aspirin taken alone, may cause renal damage. If it occurs at all this response is rare in Britain.

Some interesting questions remain after two decades of research and speculation. Do Australians have more of the disease? In some of their series it accounts for one-third of patients with fatal renal failure ${ }^{9}$ and a sixth of those presenting for dialysis and transplantation. ${ }^{10}$ Perhaps Australian doctors recognise analgesic nephropathy more often; if it really is more frequent the explanation may be that more analgesics are eaten (which they are) or that they are eaten in a hot country and are excreted in more concentrated urine; or there may be genetic predispositions. The surge of reports of associations between HLA type and certain diseases raises the possibility that interstitial renal disorders such as analgesic nephropathy may develop only in individuals with a susceptible genotype. This might explain why only a few of the many who take large quantities of compound phenacetin-containing analgesics develop renal failure.

If diagnosed before there is severe renal damage analgesic nephropathy is treatable. Renal function may be expected to improve or at least stabilise if the intake of analgesics is stopped ${ }^{2} 6$ provided that the plasma creatinine concentration is below $300-400 \mu \mathrm{mol} / 1$. At that level of renal function renal disease, whatever its aetiology, often enters an irreversible decline towards total kidney failure. ${ }^{11}$ Clearly early diagnosis is important for both the individual patient and the National Health Service-treatment of end-stage renal failure is very expensive. If analgesic nephropathy becomes less common and so receives less attention in medical journals there is a risk that it will more often be forgotten as a possible cause of renal problems. The diagnosis should be considered when any of the "typical" features are noted or when there is any uncertainty about the cause of renal failure. Among the most common clues are sterile pyuria and papillary necrosis, especially if there is also anaemia, acidosis, or sodium loss that is unusually great for the degree of renal insufficiency. If the appearances in the intravenous urogram are reported to suggest "chronic pyelonephritis" the diagnosis should be considered. Analgesic nephropathy is a good example of the validity of two popular aphorisms: "Most medical diagnoses are made by taking a good history" and "Treatable disease, even when uncommon or rare, is more important to diagnose than common but untreatable disease."

${ }^{1}$ Spühler O, Zollinger HU. Die chronisch-interstitielle nephritis. Zeitschrift für Klinische Medizin 1953;151:1-50.

2 Bell D, Kerr DNS, Swinney J, Yeates WK. Analgesic nephropathy: clinical course after withdrawal of phenacetin. Br Med F 1969 ;iii:378-82. 
${ }^{3}$ Murray RM, Lawson DH, Linton AL. Analgesic nephropathy: clinical syndrome and prognosis. $\mathrm{Br} M e d F 1971 ; \mathrm{i}: 479-82$

4 Lee HA, Davidson AR, Burston J. Analgesic nephropathy in Wessex: a clinicopathological survey. Clin Nephrol 1974;2:197-207.

${ }^{5}$ Dawbone JK, Fairley KF, Kincaid-Smith P, King WE. The association of peptic ulceration, chronic renal disease, and analgesic abuse. $Q \mathcal{F} \mathrm{Med}$ 1966 ;35:69-83.

- Cove-Smith JR, Knapp MS. Analgesic nephropathy: an important cause of chronic renal failure. $Q \mathcal{F}$ Med 1978;47:49-69.

7 Proceedings of the European Dialysis and Transplant Association, 1980. Vol 17. Tunbridge Wells: Pitman, 1981.

${ }^{8}$ Murray RM. Analgesic nephropathy: removal of phenacetin from proprietary analgesics. $\mathrm{Br} \mathrm{Med} \mathcal{f} 1972$;iv:131-2.

9 Ferguson I. How safe are analgesics? The Queensland experience. Aust NZ F Med 1974;2:603.

10 Sheil AGR, Stewart JH, Johnson JR, et al. Community treatment of end-stage renal failure by dialysis and renal transplantation from cadaver donors. Lancet 1969;ii:917-20.

11 Rutherford WE, Blondin J, Miller JP, Greenwalt AS, Vavra JD. Chronic progressive renal disease: rate of change of serum creatinine concentration. Kidney Int 1977;11:62-70.

\section{Insulin injections and infections}

Patients with diabetes develop abscesses in the thighs attributable to their insulin injections remarkably rarely. We recently reported the case of a woman in whom the cause was found to be an atypical Mycobacterium chelonei var abscessus. ${ }^{1}$ The patient used a glass syringe and disposable needles, which were used only twice but stored on the syringe in a hypochlorite solution (probably inactive as she often prepared it with boiling water, which would have driven off the chlorine). The moist environment for the syringe would have favoured the growth of mycobacteria.

The main protection from insulin injection abscesses is the addition of $0.15 \%$ metacresol and $0.06 \%$ phenol to insulin. These additives are effective in preventing growth of Staphylococcus aureus inoculated into phials of insulin at up to $10^{6}$ colony-forming units per phial and then incubated for up to 48 hours. $^{2}$ Studies in human volunteers have shown that a minimum dose of $7.5 \times 10^{6}$ Staph aureus needs to be injected intradermally to produce an abscess. ${ }^{3}$ This is a far heavier contamination than has been reported to occur naturally. ${ }^{4}$ For instance, Koivista and Felig ${ }^{4}$ found that the highest value on uncleaned human skin was $2 \times 10^{4} / \mathrm{cm}^{2}$. Nevertheless, poorly controlled diabetics have been shown to be more susceptible than non-diabetics to such infections. ${ }^{5}$

Most diabetics are asked to keep their glass insulin syringes in industrial methylated spirit, which has to be evaporated by pumping the syringe before the insulin is drawn up. Though "surgical" spirit sounds more appropriate than "industrial" spirit for keeping an insulin syringe in a safe condition, in fact the opposite is true. Leigh and Hough ${ }^{6}$ described the case of a woman whose chronic abscesses at her injection sites were attributable to the use of surgical spirit for two years. "Surgical" as opposed to "industrial" spirit contains additives such as castor oil, methyl salicylate, and diethylphthalate, which are local irritants and leave an oily residue when the alcohol evaporates.

Recent experience with repeated use of dry plastic syringes has been encouraging with regard to safety, speed, and the small dead space (which reduces wastage of insulin left in the nozzle of the syringe). Bloom and his colleagues ${ }^{7}$ found no evidence of bacterial contamination of plastic syringes used repeatedly for up to two months and kept between use in a domestic refrigerator. Hodge and his co-workers ${ }^{2}$ were equally encouraging on the basis of their experience in the United States. If such syringe-needle units are used for an individual diabetic and the caps replaced over the needle and plunger after use they seem safe when reused without chemical sterilisation until the needles get blunt or deformed or the markings wear off the syringe barrel. Provided they are kept dry syringes would not provide conditions favourable for the growth of mycobacteria. Such syringes are small to carry, easy to use, waste little insulin, and economic if not discarded after a single use (as the manufacturers recommend). No reports have yet appeared to suggest that such practice is bacteriologically unsafe. Now that agreement has been reached in Britain that it would be advantageous to change over from 20 , 40 , and 80 unit insulin to the new 100 unit strength, it will be an opportunity, when this change is effected, for more of the large population of insulin users to start to use plastic syringeneedle units at a relatively low cost.

${ }^{1}$ Jackson PG, Keen H, Noble CJ, Simmons NA. Injection abscesses in a diabetic due to Mycobacterium chelonei var abscessus. $\mathrm{Br} M e d \mathcal{F} 1980$ 281 :1105-6.

${ }^{2}$ Hodge RH Jr, Krongaard L, Sande MA, Kaiser DL. Multiple use of disposable insulin syringe-needle units. F $A M A$ 1980;244:266-7.

${ }^{3}$ Elek SD. Experimental staphylococcal infections in the skin of man. Ann NY Acad Sci 1956;65:85-90.

4 Koivista VA, Felig P. Is skin preparation necessary before insulin injection? Lancet 1978; ; 1072-3.

5 Robertson HD, Polk HC Jr. The mechanism of infection in patients with diabetes mellitus: a review of leukocyte malfunction. Surgery 1974;75: 123-8.

${ }^{6}$ Leigh DA, Hough GW. Dangers of storing glass syringes in surgical spirit. Br Med F $1980 ; 281: 541-2$.

7 Greenough A, Cockcroft PM, Bloom A. Disposable syringes for insulin injection. $\mathrm{Br} \mathrm{Med} \mathcal{F} 1979 ; \mathrm{i}: 1467-8$.

\section{Ten years of ASH}

Action on Smoking and Health (ASH) is now ten years old. Set up in 1971 under the auspices of the Royal College of Physicians as a pressure group to limit the harmful effects of smoking, it has fought hard in what has sometimes been a savage battle by supplying information to the public, doctors, media, and politicians. Some progress has been made, but much remains to be done.

Initiated by doctors, $\mathrm{ASH}$, which has a full-time staff of only five, still has a governing council that is half doctors and is proud of its "medical backbone." Most of its money comes as a grant from the Government, but it is an independent charity and has a freedom that many antismoking departments sponsored by foreign governments do not have. David Simpson, the present director, does think, however, that a British Government unit devoted entirely to dealing with smoking problems could do useful work that cannot be done either by ASH or the Health Education Council.

What has ASH achieved in the past ten years? The percentage of smokers has dropped from $52 \%$ of men and $41 \%$ of women in 1972 to $45 \%$ of men and $37 \%$ of women in 1978. This is, however, a much less dramatic drop than that achieved in some other countries-the United States, for example-and the number of cigarettes smoked per smoker has actually increased (although the average tar yield per cigarette has decreased). More importantly, attitudes have changed in those ten years: now that most people in Britain do not smoke it is slowly becoming thought normal not to smoke; most of those who do smoke mean to give up; the amount of no-smoking accommodation in trains, planes, and 